\title{
DESEMPENHO E PROPORÇÃO SEXUAL DE TILÁPIA VERMELHA SOB À INCLUSÃO DE PROBIÓTICO EM ÁGUA MESOHALINA
}

\author{
PERFORMANCE AND SEXUAL PROPORTION IN RED TILAPIA UNDER INCLUSION OF \\ PROBIOTIC IN MESOHALINE WATER
}

\author{
Marengoni, N.G. ${ }^{1 *}$, Albuquerque, D.M. ${ }^{2}$, Mota, F.L.S. ${ }^{2}$, Passos Neto, O.P. ${ }^{2}$, Silva Neto, A.A. ${ }^{2}$, \\ Silva, A.I.M. ${ }^{2}$ e Ogawa, M. ${ }^{2}$
}

${ }^{1}$ Unioeste/CCA. Rua Pernambuco, 1777. CEP 85960-000. Marechal Cândido Rondon, Paraná. Brasil. *nmarengoni@hotmail.com

${ }^{2}$ UFC/CCA. Fortaleza, Ceará. Brasil.

\section{PaLAVRAS ChaVe ADICIONAIS}

Alevinagem. Promotor de crescimento. Sistema de recirculação. Tilapicultura.

\section{RESUMO}

A tilápia vermelha é uma espécie que se adapta bem as altas salinidades sendo uma ótima candidata ao cultivo em águas mesohalinas. Os probióticos são suplementos alimentares que podem melhorar o desempenho produtivo na piscicultura. Objetivou-se avaliar o desempenho produtivo e a proporção sexual de alevinos de tilápia vermelha (Oreochromis sp., linhagem Saint Peter) em águas mesohalinas a 0,5 e 1,0\% sob inclusão de níveis crescentes de probiótico $(0,5$, 10 e $15 \mathrm{~g}$ de probiótico/kg de ração comercial). Foram usadas como unidades experimentais o sistema de recirculação de água - RAS (experimento I) e o tanque-rede - TER (experimento II) utilizando $0,5 \%$ e $1,0 \%$ de águas mesohalinas respectivamente. Utilizou-se o delineamento completamente casualizado com quatro tratamentos e três repetições. O desempenho produtivo e ajuste no fornecimento de ração foram monitorados por biometrias quinzenais com duração de 42 e 70 dias para RAS e TER, respectivamente. O peso, ganho de peso, biomassa, ganho de biomassa, conversão alimentar, ganho de peso médio diário, crescimento específico, fator de condição, sobrevivência não foram influenciados pela inclusão de probiótico $(p>0,05)$ nas duas unidades experimentais. A proporção sexual dos alevinos tanto na salinidade $0,5 \%$ quanto $1,0 \%$ não foi influenciada ( $p>0,05)$ pelos níveis de inclusão de probiótico. Ao final dos experimentos, tanto o peso corporal, quanto o

Recibido: 13-7-08. Aceptado: 16-12-08.

\section{ADDITIONAL KEYWORDS}

Growth promoter. Fingerling. Recycling system. Tilapia culture.

comprimento total dos animais apresentaram comportamento geométrico. Os níveis de probiótico utilizados na ração comercial para a alevinagem de tilápia vermelha cultivados em água mesohalina, tanto em aquário como em tanques-rede não justificam sua aplicabilidade e eficiência como promotor de crescimento nem como controlador de populações monossexo de machos.

\section{SUMMARY}

The red tilapia adapts well to the high salinity and it is a great candidate for cultivation in mesohaline water. The probiotics are food supplements that can improve the productive performance in fish farming. This work was carried out to evaluate the productive performance and the sexual proportion of the red tilapia fingerlings (Oreochromis sp., Saint Peter strain) under inclusion of increasing probiotic levels $(0,5,10$ and $15 \mathrm{~g}$ of probiotic/ $\mathrm{kg}$ of commercial ration). As experimental units were used: water recycling systems - RAS (experiment I) and cage - TER (experiment II) using $0.5 \%$ and $1.0 \%$ mesohaline water respectively. A completely randomized design with four treatments and three repetitions was used. The productive performance and adjustment in the ration supply were verified at fortnighty biometries with duration of 42 and 70 days for RAS and TER, respectively. The weight, 
weight gain, biomass, biomass gain, alimentary conversion, the mean daily weight gain, specific growth, condition factor and survival were not influenced by the inclusion of probiotic $(p>0.05)$ in the two experimental units. The sexual proportion of the fingerlings in the $0.5 \%$ and $1.0 \%$ salinity were not influenced $(p>0.05)$ for the inclusion levels of probiotic. At the end of the experiments, both body weight, and animal total length increased following a geometric model. It can be concluded that levels of commercial probiotic doesn't contribute satisfactorily to improve the performance of red tilapia reared in a recycling system (aquarium or cage) in mesohaline water.

\section{INTRODUÇÃO}

A produção mundial de tilápias e outros ciclídeos, de acordo com a FAO (2006), foi de aproximadamente $1822,745 \mathrm{t}$. A aquicultura continental no Brasil obteve uma produção de 191183,5 t, significando uma participação de 26,5\% na produção de pescado, no qual representa um acréscimo relativo de $6,4 \%$ e um crescimento de $0,4 \%$, comparado ao ano anterior. No Nordeste brasileiro, o estado do Ceará foi o maior produtor aquícola continental com aproximadamente $17177 \mathrm{t}$ de peixes produzidos em 2006, representados por 98,97\% de tilápia (IBAMA, 2008).

Segundo El-Sayed (2006) as tilápias são excelentes candidatas ao cultivo de água salgada e salobra, devido sua ampla tolerância à salinidade, porém, isto depende da espécie, tempo e método de adaptação e efeitos ambientais. A tilápia vermelha (Oreochromis sp.) é um híbrido deste gênero que se adapta bem e cresce melhor em águas salgadas e salinas que em água doce e seus descendentes toleram salinidades que variam entre 1,7\% e 3,6\%. Além disso, essa linhagem quando bem adaptada, possui um crescimento rápido, fácil manejo e boa aceitabilidade ao alimento artificial. Há uma grande necessidade do cultivo de tilápias em água salgada e salobra em regiões onde a água doce é escassa, no entanto são poucos trabalhos relacionando a este culti- vo, quando comparados aos inúmeros estudos sobre essa espécie em água doce (Basiao et al., 2005).

Em função dos problemas advindos da utilização de antibióticos, sejam eles de ordem econômica, metodológica ou sanitária, devem ser estudadas alternativas que diminuam a utilização de quimioterápicos na aqüicultura, sem perdas de produtividade e qualidade final do alimento, como por exemplo, os probióticos (Meurer, 2005).

De acordo com Silva et al. (2006) os probióticos são definidos como suplementos alimentares, contendo microorganismos vivos que afetam beneficamente o animal hospedeiro, melhorando o equilíbrio da microbiota intestinal, podendo assumir uma função alternativa de promotor de crescimento.

Os probióticos possuem efeitos na sanidade aquícola, suplementando algumas bactérias e controlando algumas doenças. Sua eficácia é estritamente dependente da quantidade e das características das cepas de microrganismos utilizadas na elaboração do aditivo alimentar. Muitas vezes estas cepas não produzem bons resultados, pois não utilizam microrganismos que atendam os requisitos para atuarem como promotor de crescimento, dos quais devem sobreviver às condições adversas do trato gastrintestinal (ação da bile e dos sucos gástricos, pancreático e entérico), não serem tóxicos e/ou patogênicos, terem capacidades antagonistas às bactérias intestinais indesejáveis, serem altamente viáveis economicamente e estáveis durante a estocagem, além de, comprovadamente, benéfico ao hospedeiro (Irianto e Austin, 2002).

Entre os efeitos positivos dos probióticos destacam-se, em geral, os diferentes mecanismos como as melhorias na qualidade de água, exclusão competitiva de bactérias nocivas por nutrientes e sítios de ligação no intestino, aumento de respostas imunológicas e benefícios no processo digestivo do hospedeiro mediante ao aporte de macro-

Archivos de zootecnia vol. 59, núm. 227, p. 404. 
TILÁPIA VERMELHA CULTIVADA EM ÁGUA MESOHALINA COM PROBIÓTICO

nutrientes, micronutrientes e enzimas digestivas (Verschuere et al., 2000).

Diversos estudos sobre probióticos foram publicados durante a última década, entre eles destacam-se os microorganismos como Bacillus spp., bactérias do ácido láctico e outras bactérias Gram-negativas (Irianto e Austin, 2002). Entretanto, as limitações metodológicas e éticas dos ensaios em animais tornaram-se difícil a compreensão dos resultados bem como sua aplicação (Balcázar et al., 2006). Nos sistemas de cultivo com altas densidades, limitada qualidade da água e falta de alimento de boa qualidade, os peixes encontram-se em constante estresse que se traduz por baixas taxas de crescimento, assim como enfermidades oportunistas (Lara-Flores et al., 2003).

O cultivo de tilápia vermelha em águas mesohalinas pode proporcionar uma fonte de renda extra nas comunidades litorâneas, entretanto são necessárias técnicas que controlem sua reprodução, pois a espécie possui características peculiares como alta capacidade de reprodução e fecundidade. Na tilapicultura, os machos crescem cerca de $30 \%$ a mais que as fêmeas, pois estas utilizam grande parte de suas reservas energéticas no processo de vitelogênese. A diferenciação sexual nos peixes envolve diversos fatores complexos, como genética da espécie, fisiologia do animal e fatores ambientais (Rouget et al., 2008). Entre os principais efeitos destaca-se a salinidade que pode ser um importante fator na diferenciação sexual, como sugerem Abucay et al. (1999). Desta forma, para conseguir uma população monossexo de machos e, com isso, maximizar a produção é importante realizar pesquisa que elucidem o processo de diferenciação sexual.

Portanto, o objetivo deste trabalho foi avaliar o desempenho produtivo e a proporção sexual de alevinos de tilápias vermelhas (Oreochromis sp.) sob inclusão de níveis crescentes de probiótico numa ração comercial utilizando diferentes águas mesohalinas (0,5 e 1,0\%) em sistemas de recirculação de água (aquários e tanquesrede).

\section{MATERIAL E MÉTODOS}

Os experimentos foram realizados no período de 13 de setembro a 22 de dezembro de 2007, no Laboratório de Recursos Aquáticos (LARAq) do Departamento de Engenharia de Pesca da Universidade Federal do Ceará (UFC), utilizando dois sistemas de recirculação de água. O experimento I, utilizando aquários foi denominado de RAS e o experimento II, utilizando tanques-rede foi definido como TER.

No experimento I, que teve duração de 48 dias, foram utilizadas 360 pós-larvas de tilápia vermelha (Oreochromis sp.) da linhagem Saint Peter com peso médio de 79 $\pm 20 \mathrm{mg}$ e 15 dias de idade, eclodidas em incubadoras artificiais com água mesohalina $0,5 \%$. O sistema de recirculação de água (RAS) foi composto por doze aquários de 20 l, cuja água foi conduzida, respectivamente, por gravidade aos filtros, mecânico e biológico. O filtro mecânico foi constituído por um material de lã acrílica e, o filtro biológico por cascalhos de ostras, onde os materiais em suspensão e restos de ração ficavam retidos. Em seguida, uma bomba submersa (Sarlo Better) com vazão de $2 \mathrm{~m}^{3} / \mathrm{h}$ recirculava a água de volta ao sistema. A água utilizada foi monitorada e a salinidade mantida a $0,5 \%$ durante todo o experimento.

Para o experimento II com duração de 70 dias, foram utilizados 360 alevinos de tilápia vermelha com peso médio de $0,30 \pm 0,10 \mathrm{~g}$, eclodidos em incubadoras artificiais com água mesohalina a 1,0\%. A unidade experimental do sistema fechado de recirculação de água (TER) constava de três tanques circulares de 1000 l e doze tanques-rede de $0,10 \mathrm{~m}^{3}$ igualmente distribuídos. A água foi drenada por gravidade e conduzida aos filtros mecânico e biológico, ambos instalados em containeres cilindros com volume de $0,22 \mathrm{~m}^{3}$ contendo, respectivamente, brita e cascalho de ostra. Uma bomba submersa

Archivos de zootecnia vol. 59, núm. 227, p. 405. 
(Sarlo Better) com vazão de $2 \mathrm{~m}^{3} / \mathrm{h}$ recirculava a água de volta ao sistema.

Utilizou-se o manejo alimentar manual com ração comercial farelada contendo $50 \%$ de proteína bruta, sendo fornecida durante todo o período $20 \%$ da biomassa para o experimento I. No experimento II a ração contendo $50 \%$ de proteína bruta foi fornecida manualmente, adotando 20\% na primeira quinzena e decorridos quinze dias $15 \%$ da biomassa dos indivíduos. A partir do primeiro mês de experimento, manteve-se o arraçoamento com $10 \%$ da biomassa com uma ração peletizada $42 \%$ de proteína bruta. O manejo alimentar teve como freqüência quatro vezes ao dia nos horários 8, 11, 14 e 17 h com duração média de 30 minutos por arraçoamento para os tanques-rede e aquários. Diariamente, os aquários foram sifonados antes de todas as alimentações para retirada das fezes e dos possíveis resíduos de ração. Cada tanque-rede possuía um sistema de comedouros feitos de garrafas PET de 2 l, onde ficavam retidas as eventuais sobras de ração.

Como probiótico utilizou-se um produto comercial (Sanolife ${ }^{\circledR}$ PRO-1), contendo no mínimo três bilhões de unidades formadoras de colônia por grama de cepas das bactérias Bacillus subtilis, Bacillus lincheniformis e Bacillus pumilus conforme o fabricante INVE do Brasil Ltda. O probiótico foi incorporado à ração com óleo vegetal um dia antes de ser ofertado aos peixes e armazenado sob refrigeração.

O delineamento experimental foi inteiramente casualizado com quatro níveis de inclusão do probiótico (0, 5, 10 e $15 \mathrm{~g}$ de probiótico/kg de ração) e três repetições. No experimento I os peixes foram distribuídos em doze aquários, considerando-se a unidade experimental um aquário com 30 pós-larvas e no experimento II os alevinos foram distribuídos em doze tanques-rede, no qual foram igualmente distribuídos em três tanques circulares de $1000 \mathrm{l}$ com 30 alevinos cada.

Registrou-se, quinzenalmente, a quanti- dade e peso total dos peixes de cada aquário e tanques-rede para determinar o ganho de peso, biomassa, sobrevivência, consumo de ração e ajustes nos arraçoamentos e, ao final do experimento foram medidos e pesados individualmente para determinação do fator de condição $(K n)$. Para a pesagem utilizou-se balança semi-analítica da marca Digimed KN 2000 com precisão de 0,01g e um erro de até $0,1 \mathrm{~g}$. Os peixes foram, ao final do experimento, insensibilizados em formalina comercial 7\% com objetivo de diminuir tempo de abate conforme Oliveira e Galhardo (2007), no qual teve um intervalo médio de morte de um a três minutos. Em seguida foram contados individualmente, pesados e medidos com paquímetro de precisão 0,01 mm e posteriormente, fixados em formalina comercial 3\% para futuras análises.

A temperatura da água foi monitorada com termômetro analógico diariamente e a salinidade mantida em 0,5 ou $1,0 \%$. Os parâmetros físico-químicos foram monitorados com potenciômetros digitais.

As determinações do teor de umidade, proteína bruta, extrato etéreo e material mineral foram realizadas em triplicata para a ração e probiótico, segundo metodologias preconizadas pela AOAC (1995). Os valores são apresentados na tabela I.

A sexagem foi realizada, ao final do cultivo, com os juvenis fixados em formalina comercial a 3\%, utilizando uma adaptação do método de esfregaço das gônadas proposto por Guerrero e Shelton (1974) e validadas por Wassermann e Afonso (2002). Esta última permitiu uma avaliação auxiliar mais rápida e precisa das estruturas gonadais.

Os valores de desempenho produtivo foram submetidos à análise de variância e em caso de diferenças significativas aplicouse o teste de Tukey. Os valores de proporção sexual foram submetidos ao teste de quiquadrado $\chi^{2}$, utilizando o programa computacional Bioestat 4.0 (Ayres et al., 2005). Para os dados de peso corporal e comprimento total foi procedida a análise de

Archivos de zootecnia vol. 59, núm. 227, p. 406. 
regressão utilizando o programa Microsoft Office Excel ${ }^{\circledR} 2007$.

\section{RESULTADOSEDISCUSSÃO}

Os valores médios de temperatura, oxigênio dissolvido (OD), condutividade e $\mathrm{pH}$ para RAS foram de $28,40^{\circ} \mathrm{C}, 5,41 \mathrm{mg} / \mathrm{l}$, $8,57 \mathrm{mS} / \mathrm{cm} \mathrm{e} 7,47$, e para TER foram $27,5^{\circ} \mathrm{C}$, 2,18 mg/l, 14,6 mS/cm e 7,27. Conforme Kubitza (2003) as tilápias suportam baixas concentrações de oxigênio dissolvido que variam entre 1,6 a $0,7 \mathrm{mg} / \mathrm{l}$ ou saturação de 10 a 20\%. Esses baixos valores de OD encontrados no presente trabalho podem estar relacionados ao aumento da biomassa dos indivíduos e a uma maior taxa de excreção. Ainda que o sistema de recirculação fechado promova uma otimização das trocas de água, recomenda-se troca parcial de água mais eficiente e/ou a utilização de um sistema de aeração suplementar para evitar a anoxia dos peixes.

Em sistemas de recirculação El-Sayed e Kawanna (2008) demonstraram que a temperatura ótima de conforto para tilápia é $28^{\circ} \mathrm{C}$. Os valores médios dos parâmetros da qualidade de água dos aquários (RAS), especificamente oxigênio dissolvido e temperatura, permaneceram dentro dos valores aceitáveis para um bom desempenho do cultivo desta espécie. Já, valores médios de OD e temperatura em TER mesmo sendo inferiores aos encontrados em RAS, mas conforme Popman e Lovshin (1996) apresentaram-se aceitáveis dentro do limite recomendado para o crescimento da espécie.
Devido aos dois sistemas serem caracterizados como recirculação fechada e com isso controlar algumas variáveis de qualidade de água, a salinidade foi monitorada diariamente em ambos sistemas e manteve-se em 0,5\% (RAS) e 1,0\% (TER) durante todo o experimento.

Analisando a tabela II, verifica-se que não houve diferenças significativas ( $\mathrm{p}>0,05)$ no desempenho produtivo tanto no sistema RAS quanto no TER. Ao final, a maioria dos parâmetros de desempenho produtivo, por exemplo, peso, biomassa e comprimento apresentaram resultados satisfatórios quando comparados com a dieta controle, embora os valores não apresentem diferenças significativas. Sugere-se um maior tempo de cultivo, outra forma de administração do produto (via água, via alimento ou as duas combinações), níveis de probiótico com intervalos maiores que $15 \mathrm{~g}$ inclusos ou misturados à ração.

Nos tanques-rede (experimento II) o peso e comprimento dos alevinos apresentaram variações entre 13,80 e 16,90 g e 8,62 e 8,95 cm, enquanto, ganho de peso diário e crescimento específico variaram entre 0,19 e $0,24 \mathrm{~g} /$ dia e 5,45 e $5,75 \% /$ dia, respectivamente os níveis de inclusão 0 a 15 g de probiótico/kg de ração. A maior sobrevivência média foi de $82,22 \%$ no nível de inclusão $0 \mathrm{~g}$. A conversão alimentar oscilou entre 1,53 a 1,58 , sendo considerado um índice para o sistema de cultivo praticado (Hisano et al., 2007).

O probiótico utilizado trata-se de uma mistura de bactérias que podem colonizar o

Tabela I. Valores médios \pm desvio padrão da composição química (\%) da ração e do probiótico utilizados. (Average \pm standard deviation of the chemical composition (\%) of ration and probiotic used).

\begin{tabular}{lcrcc}
\hline Ração & Umidade & Extrato etéreo & Proteína & Matéria mineral \\
\hline Farelada & $9,41 \pm 0,17$ & $8,14 \pm 0,29$ & $52,42 \pm 4,80$ & $7,29 \pm 0,41$ \\
Peletizada & $9,78 \pm 0,26$ & $3,42 \pm 0,18$ & $43,37 \pm 0,34$ & $6,53 \pm 0,45$ \\
Probiótico comercial & $11,91 \pm 1,43$ & $2,38 \pm 0,76$ & $45,76 \pm 2,39$ & $7,29 \pm 2,74$ \\
\hline
\end{tabular}


intestino do animal hospedeiro, diminuindo a conversão alimentar e reduzindo os custos sem alterar a produção. Em estudos comparando outro probiótico, a levedura Saccharomyces cerevisae e seus derivados em tilápia-do-Nilo, Hisano et al. (2007) verificaram que a levedura autolisada proporcionou as melhores respostas de ganho de peso a partir de um consumo maior, porém com melhor conversão alimentar em relação aos demais tratamentos, sugerindo que a levedura e derivados do seu processamento possuem quantidades satisfatórias de aminoácidos neutros, básicos e ácidos que podem conferir potencial atrato-palatabilizante nas rações suplementadas.

Possivelmente a forma na qual a bactéria foi inserida na ração e a fase na quais os alevinos de tilápia foram utilizados em RAS e TER, podem ter influenciado a não obtenção de resultados semelhantes aos

Tabela II. Valores médios e desvio padrão(sd) dos parâmetros de desempenho de tilápia vermelha alimentada com ração contendo diferentes níveis de probiótico após 42 dias de alevinagem em RAS a 0,5\% (experimento I) e 70 dias de cultivo em tanques-rede a 1,0\% (experimento II). (Average values and standard deviation of the performance parameters of red tilapia fed with ration containing different levels of probiotic after 42 days of fingerlings culture in RAS to $0.5 \%$ (experiment I) and 70 days of cultivation in cages to $1.0 \%$ (experiment II).

\begin{tabular}{|c|c|c|c|c|c|c|}
\hline \multirow[t]{2}{*}{ Parâmetro } & \multicolumn{4}{|c|}{ Nível de inclusão de probiótico (g/kg) } & \multirow[b]{2}{*}{ CV\% } & \multirow[b]{2}{*}{$\mathrm{p}$} \\
\hline & 0 & 5 & 10 & 15 & & \\
\hline \multicolumn{7}{|c|}{ Experimento I (RAS) } \\
\hline Peso (g) & $1,21 \pm 0,13$ & $1,29 \pm 0,20$ & $1,29 \pm 0,02$ & $1,45 \pm 0,19$ & 7,54 & 0,1707 \\
\hline Ganho de peso (g) & $1,13 \pm 0,13$ & $1,21 \pm 0,20$ & $1,21 \pm 0,02$ & $1,37 \pm 0,19$ & 8,02 & 0,3467 \\
\hline Biomassa (g) & $28,12 \pm 2,73$ & $32,03 \pm 4,44$ & $30,98 \pm 4,46$ & $34,51 \pm 2,37$ & 8,42 & 0,2658 \\
\hline Ganho de biomassa (g) & $25,75 \pm 2,73$ & $29,66 \pm 4,44$ & $28,61 \pm 4,46$ & $32,14 \pm 2,37$ & 9,10 & 0,2659 \\
\hline Comprimento $(\mathrm{cm})$ & $3,93 \pm 0,07$ & $4,10 \pm 0,2$ & $4,07 \pm 0,01$ & $4,15 \pm 0,27$ & 2,35 & 0,5347 \\
\hline GPMD $^{1}(g / d i a)$ & $0,03 \pm 0,01$ & $0,03 \pm 0,01$ & $0,03 \pm 0$ & $0,03 \pm 0,01$ & 10,94 & 0,3634 \\
\hline $\mathrm{CE}^{2}(\% / \mathrm{dia})$ & $6,49 \pm 0,26$ & $6,63 \pm 0,36$ & $6,65 \pm 0,04$ & $6,91 \pm 0,31$ & 2,62 & 0,3556 \\
\hline Fator de condição & $1,76 \pm 0,10$ & $1,70 \pm 0,02$ & $1,75 \pm 0,03$ & $1,70 \pm 0,02$ & 2,03 & 0,3797 \\
\hline Consumo de $r$ & $51,07 \pm 4,26$ & $53,87 \pm 5,58$ & $55,72 \pm 1,94$ & $57,21 \pm 1,69$ & 4,86 & 0,2859 \\
\hline Conv & $1,99 \pm 0,05$ & $1,82 \pm 0,10$ & $1,98 \pm$ & $1,79 \pm 0,11$ & 7,21 & 0,3704 \\
\hline & $82,22 \pm 5,09$ & $3,33 \pm 8,82$ & $80,00 \pm 12,02$ & $76,67 \pm 0,00$ & 3,65 & 0,7474 \\
\hline \multicolumn{7}{|c|}{ Experimento II (TER) } \\
\hline Peso (g) & $13,80 \pm 2,82$ & $15,25 \pm 0,58$ & $15,10 \pm 1,93$ & $16,90 \pm 2,02$ & 8,33 & 0,3694 \\
\hline Ganho de peso (g) & $13,50 \pm 2,82$ & $14,95 \pm 0,58$ & $14,80 \pm 1,93$ & $16,60 \pm 2,02$ & 8,50 & 0,3694 \\
\hline Biomassa (g) & $341,62 \pm 79,80$ & $316,14 \pm 69,48$ & $366,86 \pm 46,19$ & $399,53 \pm 54,25$ & 10,01 & 0,5314 \\
\hline Ganho de biomassa (g) & $332,62 \pm 79,80$ & $307,14 \pm 69,48$ & $357,86 \pm 46,19$ & $390,53 \pm 54,25$ & 10,27 & 0,5054 \\
\hline Comprimento (cm) & & $8,95 \pm 0,45$ & $8,69 \pm 0,47$ & $8,90 \pm 0,23$ & 1,83 & 1,0000 \\
\hline GPMD $^{1}$ (g/dia) & $0,19 \pm 0,28$ & $0,21 \pm 0,01$ & $0,21 \pm 0,03$ & $0,24 \pm 0,03$ & 8,49 & 0,366 \\
\hline $\mathrm{CE}^{2}(\% / \mathrm{dia})$ & & & & & 2,21 & 0,3600 \\
\hline Fator de cond & & & & $1,99 \pm 0,05$ & 3,64 & 0,5980 \\
\hline Consur & $515,78 \pm 66,00$ & $472,05 \pm 94,23$ & $553,68 \pm 55,98$ & $590,00 \pm 38,14$ & 9,50 & 0,2384 \\
\hline Conversão alimentar & $1,58 \pm 0,20$ & $1,54 \pm 0,07$ & $1,55 \pm 0,06$ & $1,53 \pm 0,27$ & 9,69 & 0,9888 \\
\hline Sobrevivência (\%) & $82,22 \pm 5,09$ & $68,89 \pm 13,47$ & $81,11 \pm 5,09$ & $81,11 \pm 5,09$ & 8,06 & 0,7474 \\
\hline
\end{tabular}

${ }^{1}$ Ganho de peso médio diário. ${ }^{2}$ Crescimento específico. CV\%: coeficiente de variação. p: probabilidade. Não houve diferença significativa a $5 \%$.

Archivos de zootecnia vol. 59, núm. 227, p. 408. 
encontrados por Hisano et al. (2007). Entretanto, Lara-Flores et al. (2003), utilizando um probiótico comercial contendo uma mistura de bactérias de Streptococcus faecium e Lactobacillus acidophilus e a levedura Saccharomyces cerevisae em tilápia-do-Nilo (Oreochromis niloticus) obtiveram resultados significativos no desempenho dos peixes em relação ao tratamento controle, independentemente do probiótico ser uma bactéria ou uma levedura.

Os resultados de desempenhos produtivos encontrados neste trabalho estão de acordo com Meurer et al. (2006), que utilizando a levedura Saccharomyces cerevisiae como probiótico para as fases iniciais do cultivo de tilápia-do-Nilo (Oreochromis niloticus) não verificaram efeito positivo, porém influenciaram nos valores de coliformes fecais intestinais dos peixes. Já, Gunther e Jiménez-Montealegre (2004), encontraram diferenças $(\mathrm{p}<0,01)$ na conversão alimentar e ganho de peso nas tilápias submetidas ao estresse e alimentadas com probiótico composto por Bacillus subtilis.

Segundo Irianto e Austin (2002), LaraFlores et al. (2003) e Hisano et al. (2007) os probióticos podem funcionar como imunoestimulantes e destacam-se pelo melhor aproveitamento do alimento, diminuindo a conversão alimentar, reduzindo, portanto, os custos de produção. Isso pode ser explicado pelo fato que os microorganismos são capazes de decompor as moléculas dos macronutrientes e transformá-las em aminoácidos, ácidos graxos e carboidratos simples.

Os efeitos positivos dos promotores de crescimento no desempenho produtivo em animais nem sempre são evidenciados, isso pode estar relacionado com as características de cada probiótico utilizado em diversas espécies, o tipo de ingrediente das dietas ou nível de estresse aos quais os animais estão submetidos. Aly et al. (2008) quando compararam o efeito potencial de duas doses de Bacillus pumilus e um probiótico comercial (Organic Green ${ }^{\mathrm{TM}}$ ) após um e dois meses de aplicação numa provável resposta imunológica, sobrevivência, crescimento e resistência na tilápia-do-Nilo infectada por Aeromonas hidrophila, reportaram que as baixas doses destes probióticos induziram significantes incrementos de peso após dois meses de aplicação do produto.

A sobrevivência foi superior a 80\%, para a maioria dos tratamentos (tabela II), considerada um ótimo resultado para tilápia vermelha, na fase de alevinagem, cultivada em sistema de recirculação de água mesohalina. Gunther e Jiménez-Montealegre (2004) também sugeriram que a adição dos probióticos possui efeitos adversos e que em alguns casos pode aumentar a sobrevivência no cultivo e o crescimento específico dos animais é conseqüência de sua sanidade. Neste estudo, os excelentes valores de conversão alimentar, especialmente para o experimento II (tabela II), e os indicadores de qualidade de água confirmam, ainda que em sistema de recirculação, apesar da leve queda nos teores de oxigênio dissolvido, a interação dos microorganismos nos filtros biológicos para manter a higidez do sistema.

Embora o presente trabalho não apresente diferenças significativas entre os níveis de inclusão do probiótico, as bactérias que compõem os probióticos quando colonizam o intestino dos peixes podem diminuir o consumo de ração, e com isso ser um produto ecologicamente correto sem reduzir a produtividade. Isso resultará numa menor quantidade de matéria orgânica eliminada ao meio ambiente, diminuindo assim, os possíveis efluentes oriundos da aquicultura. Além disso, podem proporcionar efeitos positivos ao interagirem com o solo e a água, controlando o aparecimento de patologias (Gunther e Jiménez-Montealegre, 2004).

O cultivo de peixes em água mesohalina $(0,5 \%$ e $1,0 \%)$ neste estudo somado ao sistema de recirculação de água foram, possivelmente, promotores de um ambiente de estresse, proporcionando uma resposta 
compensatória do efeito do probiótico sobre o desempenho produtivo. Taoka et al. (2006), verificando os efeitos dos probióticos nas respostas imunológicas não-específicas, tolerância ao estresse e resistência introduzidos por diferentes probióticos comerciais na tilápia-do-Nilo demonstraram que os tratamentos que continham probióticos apresentaram valores significativos de hemoglobina no sangue quando compa- rados ao tratamento controle após serem submetidos ao teste de salinidade. Os autores sugerem que os probióticos podem afetar beneficamente as condições de estresse nos peixes no aspecto sobrevivência, concentração protéica no muco da pele, embora os resultados do experimento não sejam claros.

A salinidade pode ser considerada um fator estressante, pois apesar do acasa-

Tabela III. Números de peixes analisados, valores médios e desvio padrão (sd) de peso corporal (PC), comprimento total (CT) e fator de condição (Kn) de alevinos de tilápia vermelha, por sexo, ao final dos experimentos. (Numbers of fish analysed, average and standard deviation (sd) of body weight (PC), total length (CT) and the condition factor (Kn) of red tilapia fingerlings for sex, at the end of the experiments).

\begin{tabular}{|c|c|c|c|c|c|c|c|}
\hline \multirow[t]{2}{*}{ Parâmetro } & \multicolumn{4}{|c|}{ Nível de inclusão de probiótico (g/kg) } & \multirow[t]{2}{*}{ Média } & \multirow[t]{2}{*}{ sd } & \multirow[t]{2}{*}{ CV\% } \\
\hline & 0 & 5 & 10 & 15 & & & \\
\hline \multicolumn{8}{|c|}{ Experimento I (RAS 0,5\%) } \\
\hline$\%$ machos & 54,69 & 48,48 & 50,00 & 57,14 & 52,58 & 4,03 & 7,66 \\
\hline \% fêmeas & 45,31 & 51,52 & 50,00 & 42,86 & 47,42 & 4,03 & 8,50 \\
\hline $\mathrm{N}^{\circ}$ peixes analisados & 64,00 & 66,00 & 62,00 & 70,00 & - & - & - \\
\hline$\chi^{2}$ & 0,39 & 0,02 & 0,00 & 1,16 & - & - & - \\
\hline $\mathrm{p}$ & 0,53 & 0,90 & 1,00 & 0,28 & - & - & - \\
\hline PC macho $(\mathrm{g})$ & $1,33 \pm 0,27$ & $1,37 \pm 0,18$ & $1,3 \pm 0,14$ & $1,36 \pm 0,34$ & 1,34 & 0,03 & 2,08 \\
\hline CT macho $(\mathrm{cm})$ & $3,76 \pm 0,45$ & $4,03 \pm 0,20$ & $3,83 \pm 0,22$ & $3,89 \pm 0,32$ & 3,88 & 0,12 & 3,00 \\
\hline PC fêmea $(g)$ & $1,32 \pm 0,08$ & $1,43 \pm 0,34$ & $1,51 \pm 0,25$ & $1,64 \pm 0,31$ & 1,47 & 0,14 & 9,25 \\
\hline CT fêmea (cm) & $3,84 \pm 0,14$ & $4,02 \pm 0,31$ & $4 \pm 0,32$ & $4,07 \pm 0,28$ & 3,98 & 0,10 & 2,55 \\
\hline Kn macho & $2,08 \pm 0,09$ & $1,95 \pm 0,01$ & $2,6 \pm 0,95$ & $2,11 \pm 0,07$ & 2,19 & 0,29 & 13,13 \\
\hline Kn fêmea & $2,07 \pm 0,11$ & $1,98 \pm 0,04$ & $2,62 \pm 0,94$ & $2,16 \pm 0,11$ & 2,21 & 0,29 & 12,92 \\
\hline$\chi^{2}$ (associação) & \multicolumn{4}{|c|}{1,31} & & & \\
\hline \multirow[t]{2}{*}{$\mathrm{p}$} & \multicolumn{4}{|c|}{0,73} & & & \\
\hline & \multicolumn{4}{|c|}{ Experimento II (TRE 1,0\%) } & & & \\
\hline$\%$ machos & 64,71 & 48,21 & 57,97 & 57,14 & 57,01 & 6,77 & 11,88 \\
\hline \% fêmeas & 35,29 & 51,79 & 42,03 & 42,86 & 42,99 & 6,77 & 15,76 \\
\hline $\mathrm{N}^{\circ}$ peixes analisados & 68,00 & 56,00 & 69,00 & 70,00 & - & - & - \\
\hline$\chi^{2}$ & 5,31 & 0,02 & 1,45 & 1,16 & - & - & - \\
\hline $\mathrm{p}$ & 0,02 & 0,89 & 0,23 & 0,28 & - & - & - \\
\hline PC macho $(\mathrm{g})$ & $18,77 \pm 6,39$ & $20,65 \pm 4,19$ & $20,9 \pm 4,39$ & $20,07 \pm 0,51$ & 20,10 & 0,95 & 4,72 \\
\hline CT macho (cm) & $8,91 \pm 1,01$ & $9,28 \pm 0,86$ & $9,24 \pm 0,83$ & $9 \pm 0,30$ & 9,11 & 0,18 & 1,97 \\
\hline PC fêmea (g) & $10,78 \pm 0,85$ & $12,9 \pm 3,18$ & $12,67 \pm 2,67$ & $14,71 \pm 3,06$ & 12,76 & 1,61 & 12,58 \\
\hline CT fêmea (cm) & $7,57 \pm 0,26$ & $7,93 \pm 0,67$ & $7,85 \pm 0,62$ & $8,22 \pm 0,57$ & 7,89 & 0,27 & 3,43 \\
\hline $\mathrm{Kn}$ macho & $2,33 \pm 0,05$ & $2,34 \pm 0,08$ & $2,21 \pm 0,08$ & $2,31 \pm 0,09$ & 2,30 & 0,06 & 2,61 \\
\hline Kn fêmea & $2,26 \pm 0,09$ & $2,3 \pm 0,10$ & $2,14 \pm 0,22$ & $2,32 \pm 0,09$ & 2,26 & 0,08 & 3,71 \\
\hline$\chi^{2}$ (associação) & \multicolumn{4}{|c|}{3,43} & & & \\
\hline $\mathrm{p}$ & \multicolumn{4}{|c|}{0,33} & & & \\
\hline
\end{tabular}

CV\%: coeficiente de variação; p: probabilidade.

Archivos de zootecnia vol. 59, núm. 227, p. 410. 
lamento, fertilização, incubação e eclosão das larvas terem sido realizadas em água mesohalina, com a mesma salinidade utilizada na alevinagem, esta é uma espécie de água doce e precisa de um maior aporte de energia para realizar o processo de regulação osmótica. Contudo, a salinidade faz com que o efeito hierárquico seja minimizado e assim, proporcione um melhor desempenho e sobrevivência.

No experimento RAS foram analisados 262 alevinos, sendo 138 machos e 124 fêmeas (tabela III). Os valores mínimos e máximos de machos encontrados foram 31 e 40 para os tratamentos 10 e 15, e de fêmeas 29 e 31 para os níveis de inclusão de 0 e 5 , respectivamente. Não houve diferenças significativas entre a proporção sexual no experimento I em todos os níveis de inclusão de probiótico $\left(\chi^{2}=0,391 ; \mathrm{gl}=1 ; \mathrm{p}=0,532\right.$ para T0; $\chi^{2}=0,015 ; \mathrm{gl}=1 ; \mathrm{p}=0,902$ para T5; $\chi^{2}=$ 0,$000 ; \mathrm{gl}=1 ; \mathrm{p}=1$ para $\mathrm{T} 10$ e $\chi^{2}=1,157 ; \mathrm{gl}=$ $1 ; \mathrm{p}=0,282$ para $\mathrm{T} 15)$.

Ao todo foi analisado o sexo fenotípico de 263 peixes no experimento TER, nos quais a população de machos foi de 151 e de fêmeas 112, não apresentando diferenças significativas para os diferentes níveis de inclusão do produto comercial Sanolife ${ }^{\circledR}$ PRO-1, conforme o teste qui-quadrado $\chi^{2}$ (tabela III). Resultados semelhantes foram observados por Abucay et al. (1999) não encontrando efeito dos diferentes níveis de salinidade (1,13 a 2,67\%) na proporção sexual em tilápia-do-Nilo.

Os valores médios de temperatura neste estudo foram de 28,40 e $27,5^{\circ} \mathrm{C}$, respectivamente para RAS e TER e não devem ter contribuído para um desvio na proporção sexual dos alevinos de tilápia vermelha (tabela III). Rouget et al. (2008) observaram que altas temperaturas podem modificar o processo normal de diferenciação sexual da tilápia-do-Nilo conjuntamente à salinidade, porém, não foi descrito, ao certo, o efeito de interação da entre salinidade e mecanismo de diferenciação sexual. Toguyéni et al. (2002), estudando a influência sexual feno- típica e genotípica e proporção sexual na performance do crescimento da tilápia-doNilo, sugeriram que a proporção sexual pode ser alterada e que a espécie é sensível às interações sociais. Em estudos sobre o processo de diferenciação sexual em alguns animais o mecanismo pode ser afetado por fatores genéticos e ambientais como, $\mathrm{pH}$, altas temperaturas da água ou ação desta no metabolismo hormonal dos peixes, influenciando tendenciosamente, tanto para machos quanto para fêmeas.

Ao final dos experimentos, tanto o peso corporal, quanto o comprimento total dos animais apresentaram comportamento geométrico expresso pelas equações de regressão $\mathrm{Y}=0,0369 \mathrm{x}^{2,5754}\left(\mathrm{R}^{2}=0,84\right)$ e $\mathrm{Y}=$ $0,0206 x^{3,0064}\left(R^{2}=0,96\right)$, respectivamente para machos e fêmeas do sistema RAS e $\mathrm{Y}=0,0162 \mathrm{x}^{3,1575}\left(\mathrm{R}^{2}=0,97\right)$ e $\mathrm{Y}=0,0146 \mathrm{x}^{3,2054}$ $\left(R^{2}=0,94\right)$, respectivamente para machos e fêmeas do sistema TER (figura 1). Os valores de coeficiente de variação para peso corporal e comprimento total dos peixes nos dois experimentos foram baixos, discordando dos resultados encontrados por Borges et al. (2005), em estudos de populações monossexo de tilápia em altas temperaturas. Estes resultados são importantes e podem interferir no desempenho produtivo, no aspecto do crescimento heterogêneo, pois o estabelecimento de hierarquia entre os peixes maiores sobre os menores promove uma desuniformidade do lote. Então, nas próximas fases de cultivo serão necessárias seleções entre os peixes, com um descarte dos menores, tendo como conseqüência um menor lucro para o produtor.

\section{CONCLUSÕES}

Os níveis do probiótico comercial Sanolife ${ }^{\circledR}$ PRO-1 utilizado na ração comercial para a fase de alevinos cultivados em água mesohalina com salinidade 0,5 ou 1,0\% não justificam sua aplicabilidade como promotor de crescimento e diferenciador do 


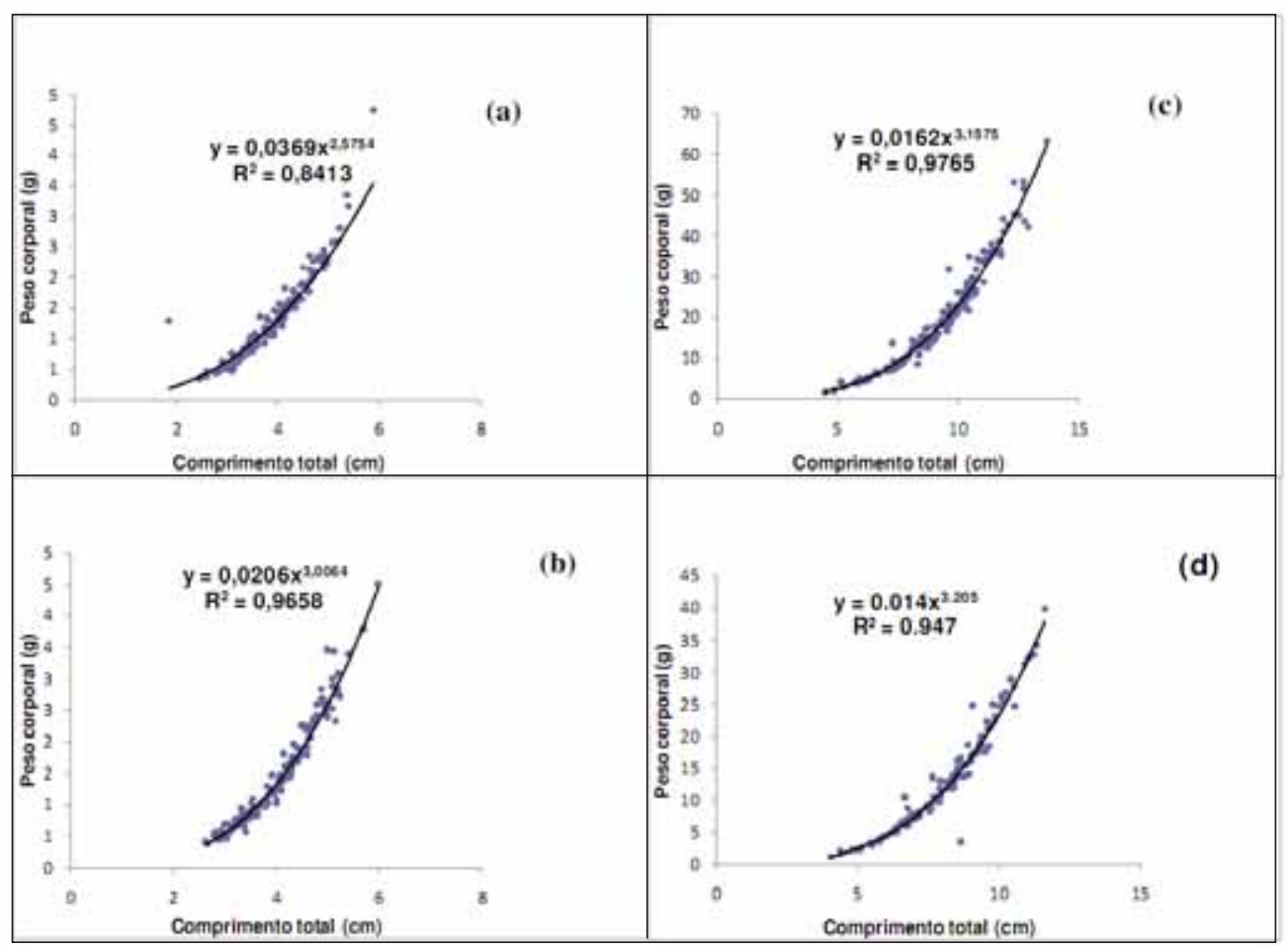

Figura 1. Curvas de regressão para peso corporal e comprimento total para macho (a) e fêmea (b), e macho (c) e fêmea (d) de tilápia vermelha nas salinidades 0,5 \% e 1,0\%, respectivamente. (Regression curves for body weight and total length of red tilapia male (a) and female (b), and male (c) and female (d) in salinities $0.5 \%$ and $1.0 \%$, respectively).

sexo fenotípico, especialmente no sistema RAS.

Sugere-se a não utilização do probiótico comercial estudado, contendo a bactéria do gênero Bacillus, nas dietas dos alevinos de tilápia vermelha cultivados em sistemas de recirculação de água mesohalina. Meios alternativos e eficazes, bem como outras formas de inclusão na água e alimento, for-

\section{BIBLIOGRAFIA}

Abucay, J.S., Mair, G.C., Skibinski, D.O.F. and Beardmore, J.A. 1999. Environmental sex determination: the effect of temperature and salinity on sex ratio in Oreochromis niloticus L. Aquaculture, 173: 219-234. ma indireta na qual o probiótico pode contribuir no meio ambiente e na microbiota das tilápias cultivadas, também devem ser esclarecidos em futuros estudos. Recomenda-se mais pesquisas que procurem elucidar o mecanismo de diferenciação sexual em demais salinidades e com outros probióticos, para proporcionar avanços tecnológicos relacionados à produção desta espécie.

Archivos de zootecnia vol. 59, núm. 227, p. 412.

Aly, S.M., Mohamed, M.F. and John, G. 2008. Effects of probiotics on the survival, growth and challenge infection in Tilapia nilotica (Oreochromis niloticus). Aquac. Res., 39: 647656. 


\section{TILÁPIA VERMELHA CULTIVADA EM ÁGUA MESOHALINA COM PROBIÓTICO}

AOAC. 1995. Official methods of analysis. Association of Official Analytical Chemists. Arlington, 2: 1-30.

Ayres, M., Ayres, M. Jr., Ayres, D.L. e Santos, A.S. 2005. Biostat. Versão 4.0, Sociedade civil Mamirauá; MCT-CNPq. Belém, Pará. Brasil.

Balcázar, J.L., De Blas, I., Ruiz-Zarzuela, I., Cunningham, D., Vendrell, D. and Múzquiz, J.L. 2006. The role of probiotics in aquaculture. Vet. Microbiol., 114: 173-186.

Basiao, Z.O., Enguia, R.V. and Doyle, R.W. 2005. Growth response of Nile tilapia fry to salinity stress in the presence of an "internal reference" fish. Aquac. Res., 36: 712-720.

Borges, A.M., Moretti, J.O.C., McManus, C. e Mariante, A.S. 2005. Produção de populações monossexo macho de tilápia-do-Nilo da linhagem Chitralada. Pesqui. Agropecu. Bras., 40: 153159.

El-Sayed, A.F.M. 2006. Tilapia culture in salt water: environmental requirements, nutritional implications and economic potentials. Em: VIII Simposium Internacional de Nutrición Acuícola. Universidad Autónoma de Nuevo León. Anais... Monterrey. Monterrey.

El-Sayed, A.F.M. and Kawanna, M. 2008. Optimum water temperature boosts the growth performance of Nile tilapia (Oreochromis niloticus) fry reared in a recycling system. Aquac. Res., 39: 670-672.

FAO. 2006. The state of world fisheries and aquaculture 2006. Food and Agriculture Organization of the United Nations. Rome.

Guerrero, R.D. and Shelton, W.L. 1974. An acetocarmine squash method of sexing juvenile fishes. Prog. Fish. Cult., 36: 56.

Gunther, J. y Jiménez-Montealegre, R. 2004. Efecto del probiótico Bacillus subtilis sobre el crecimiento y alimentación de tilapia (Oreochromis niloticus) y langostino (Macrobrachium rosenbergii) en laboratorio. Rev. Biol. Trop., 52: 437-943.

Hisano, H., Narváez-Solarte, W.V., Barros, M.M. e Pezzato, L.E. 2007. Desempenho produtivo de alevinos de tilápia-do-Nilo alimentados com levedura e derivados. Pesqui. Agropecu. Bras., 42: 1035-1042.

IBAMA. 2008. Instituto Brasileiro do Meio Ambiente e dos Recursos Naturais Renováveis. 2008. Estatística da pesca no Brasil: Grandes regiões e unidades da federação. IBAMA. Brasil. 174 $\mathrm{pp}$.

Irianto, A. and Austin, B. 2002. Probiotics in aquaculture. J. Fish. Dis., 25: 633-642.

Kubtiza, F. 2003. Qualidade da água no cultivo de peixes e camarões. Fernando Kubitza. Jundiaí. São Paulo. 229 pp.

Lara-Flores, M., Olvera-Novoa, M.A., GuzmánMendéz, B.E. and López-Madrid, W. 2003. Use of bacteria Streptococcus faecium and Lactobacillus acidophilus, and the yeast Saccharomyces cerevisiae as growth promoters in Nile tilapia(Oreochromis niloticus). Aquaculture, 216: 193-201.

Meurer, F. 2005. Levedura (Saccharomyces cerevisiae) como probiótico para as fases iniciais do cultivo da tilápia do Nilo (Oreochromis niloticus). Tese (Doutorado em Zootecnia). Universidade Estadual de Maringá. Maringá, PR. $85 \mathrm{pp}$.

Meurer, F., Hayashi, C., Costa, M.M., Mauerwerk, V.L. e Freccia, A. 2006. Utilização da Saccharomyces cerevisiae como probiótico para tilápias do Nilo (Oreochromis niloticus) durante o período de reversão sexual submetidas a um desafio sanitário. Rev. Bras. Zootecn., 35: 1881-1886.

Oliveira, R.F. e Galhardo, L. 2007. Sobre a aplicação do conceito de bem-estar a peixes teleósteos e implicações para a piscicultura. Rev. Bras. Zootecn., 36: 77-86.

Popman, T.J. and Lovshin, L.L. 1996. World wide prospects for commercial production of tilapia. International Center to Aquaculture and Aquatic Environments. Alabama. (Research and Development Series, 41). 23 pp.

Rouget, C., Prignon, C., Kengne, C.V. and Mélard, C. 2008. Effect of high temperature during embryogenesis on the sex differentiation process in the Nile tilapia, Oreochromis niloticus. Aquaculture, 276: 205-208.

Silva, C.A., Hoshi, E.H., Pacheco, G.D. e Briganó, M.V. 2006. Avaliação de probióticos (Pediococcus acidilactici e Bacillus subtilis) após o desmame e efeitos no desempenho dos leitões. Semina, 27: 133-140.

Taoka, Y., Maeda, H., Jo, J.Y., Kim, S.M., Park, S.I., Yoshikawa, T. and Sakata, T. 2006. Use of live and dead probiotic cells in tilapia Oreochromis niloticus. Fish. Sci., 72: 755-766.

Archivos de zootecnia vol. 59, núm. 227, p. 413. 
MARENGONI, ALBUQUERQUE, MOTA, PASSOS NETO, SILVANETO, SILVAE OGAWA

Toguyéni, A., Fauconneau, B., Fostier, A., Abucay, J., Mair, G. and Bairoller, J.B. 2002. Influence of sexual phenotype and genotype, and sex ratio on growth performances in tilapia, Oreochromis niloticus. Aquaculture, 207: 249-261.

Verschuere, L., Rombaut, G., Sorgeloos, P. and Verstraete, W. 2000. Probiotic bacteria as biological control agents in aquaculture. Rev. Microbiol. Mol. Biol., 64: 655-671.

Wassermann, G.J. and Afonso, L.O.B. 2002. Validation of the aceto-carmine technique for evaluating phenotypic sex in Nile tilapia (Oreochromis niloticus) fry. Cienc. Rural, 32: 133-139.

Archivos de zootecnia vol. 59, núm. 227, p. 414. 